\title{
Analysis of the downlink saturation throughput of an asymmetric IEEE 802.11n-based WLAN
}

Mohammad Soleymani, Behrouz Maham, Farid Ashtiani

- School of Engineering

\section{Abstract}

Frame aggregation (FA) mechanisms improve the throughput of WLANs. In this paper, the effect of the FA mechanism on the throughput of wireless local area networks (WLANs) has been investigated. To this end, we propose an analytical model in order to analyze an IEEE 802.11n network comprised of an access point (AP) and several conventional nodes (CNs), all in the coverage area of each other. With respect to the heavier download traffic compared to the upload one, in our scenario, only the AP uses an FA mechanism and the other nodes use the basic IEEE 802.11 standard. In our proposed analytical model, the maximum downlink (DL) throughput is derived. Regarding the asymmetry among nodes, our analytical model consists of two different queueing networks: one for the AP and the other one for CNs. We verify the accuracy of our analytical results by simulations, i.e., less than $5 \%$ mismatch between the analytical and simulation results. We show that there is a tradeoff between the DL saturation throughput and performance of CNs. In other words, the FA improves the AP saturation throughput at the cost of a little degradation of the performance for CNs.

Original language English

Title of host publication

Publisher

ISBN (Electronic)

State

Event

Other

Other

Country

City
2016 IEEE International Conference on Communications, ICC 2016

$\underline{\text { Institute of Electrical and Electronics Engineers Inc. }}$

9781479966646

Published - Jul 122016

2016 IEEE International Conference on Communications, ICC 2016 - Kuala Lumpur, Malaysia

2016 IEEE International Conference on Communications, ICC 2016

Malaysia

Kuala Lumpur 
Soleymani, M., Maham, B., \& Ashtiani, F. (2016). Analysis of the downlink saturation throughput of an asymmetric IEEE 802.11n-based WLAN. In 2016 IEEE International Conference on Communications, ICC 2016. [7511453] Institute of Electrical and Electronics Engineers Inc.. DOI: $\underline{10.1109 / I C C .2016 .7511453}$ 